\title{
La Incompetencia Cervical Uterina como causa de Aborto Habitual (1)
}

\author{
DR. RENE DIAZ CORREA (2)
}

A. Z. de A. 28 años. Casada hace 9 años. Antecedentes de pielitis y amigdalitis en la niñez. Presentó la menarca a los 15 años con ciclo menstrual normal en el período prematrimonial. Tendencia a la polimenorrea en los últimos años. Grávida 4, Para 1.- Los abortos fueron de 5,3 y $4 \frac{1}{2}$ meses respectivamente.

El primer aborto fué atendido en Bogotá. El segundo tuvo lugar en Medellín el 8 de Octubre de 1955 . Requirió raspado complementario bajo anestesia. No se conocen detalles especiales en estos primeros abortos, aunque es digno de tenerse en cuenta el tiempo de evolución de la gestación cuando se presentaron.

Consultó por primera vez el 8 de Enero de 1957. Su última menstruación había tenido lugar el 24 de Octubre de 1956 y la reacción biológica de gestación ordenada en esa ocasión fué positiva. Se prescribió Dietilestilbestrol en dosis progresivas, empezando con 10 mgs., diariamente. Se complementó la terapia con tranquilizantes dada la inestabilidad emocional de la paciente y franco pesimismo en cuanto al futuro de sus embarazos. Se tranquilizó y estimuló a la paciente en forma amplia, ofreciéndole toda clase de apoyo profesional.

La evolución de su tercera gestación fué relativamente normal hasta los cuatro meses cuando se presentó pequeña metrorragia oscura, intermitente y sensación de malestar pélvico. Se complementó la medicación con altas dosis de Progesterona parenteral. El 24 de abril de 1957 a los cuatro meses y medio de embarazo presentó expulsión del huevo intacto, en forma fácil, con feto y vivo y la impresión objetiva de dilatación pasiva del cuello uterino.

Debido al impacto emocional ocasionado por los abortos anteriores, con feto en buen estado de desarrollo y vivo, la pareja optó por medidas anticoncepcionales al menos temporalmente mientras se desvanecía un poco el recuerdo y la desconfianza.

(1) Trabajo leído en la Asociación Antioqueña de Obstetricia y Ginecología, en la sesión del 2 de Ägosto de 1960.

(2) Del Departamento de Ginecología. Facultad de Medicina de la Univerdad de Antioquia. 
Una nueva revisión genital practicada el 24 de Octubre de 1958 confirmó ampliamente la sospecha de incompetencia cervical. Se explicó la condición anormal del cuello uterino a los cónyuges y la posibilidad de remediarla, mediante ligadura circular previa o durante el embarazo, según el procedimiento de Shirodkar modificado por Green Armytage. Se estimulan a probar suerte con un nuevo embarazo.

El 23 de Octubre de 1959 la paciente tiene su última menstruación.

El 10 de Diciembre de 1959 se procedió al cerclaje del cuello a la altura del orificio interno con Nylon $\mathrm{N}^{\circ} 4$ en la cuarta semana de gestación comprobada clínica y biológicamente.

El embarazo evoluciona en forma comparativamente menos ruidosa que los tres anteriores. La ligadura fué bien tolerada, no obstante los cabos de pulgada de longitud dejados en la superficie anterior del cuello. Solo el 21 de Junio, es decir a los $71 \frac{1}{2}$ meses de gestación se presentó hipermotilidad fetal y algunas contracciones uterinas que se atribuyeron a reactivación de la inestabilidad emocional de la madre, motivada por problemas de índole conyugal. Se envió en consulta al obstetra, el cual insinuó retirar la ligadura al completar el octavo mes por considerar viable el feto y teniendo en cuenta dos posibles connatos de iniciación de parto.

Se retiró la ligadura cervical en el consultorio, en forma relativamente fácil el 12 de Julio de 1960. Cuarenta horas después y en forma precipitada, sin contracciones dolorosas y sin necesidad de anestesia se presentó el parto con feto femenino de 2.800 gramos, el cual respiró y lloró inmediatamente. El obstetra observó escaso líquido amniótico ( no por pérdida previa) de color verdoso, posiblemente debido a expulsión y disolución de meconio. La niña presentó atelectasia total del pulmón izquierdo y parcial del derecho comprobada clínica y radiológicamente, de la cual se repuso totalmente en 72 horas, mediante tratamiento apropiado.

\section{CONSIDERACIONES ETIOLOGICAS CLINICAS Y TERAPEUTICAS}

La incompetencia cervical uterina como causa de aborto en el segundo trimestre de la gestación y de parto prematuro, ha sido una adquisición obstetro-ginecológica de los últimos 12 años.

Corresponde el gran ginecólogo francés Palmer y a su asociado Lacomme la primicia clínica y quirúrgica lograda en un caso publicado en 1948. En 1950 los norteamericanos Lash and Lash (1) describieron una técnica similar a la de Palmer, que trataba de corregir la falla tisular del cuello uterino. Posteriormente fueron divulgados nuevas contribuciones encaminadas a precisar ésta entidad 
por Shirodkar de la India y más recientemente por Green Armytage (5) de Inglaterra, MacDonald (4) de Australia y los americanos Barter (2) (3) y Page.

\section{FRECUENCIA}

Comparativamente con el número de pacientes que presentan abortos en el primer trimestre, la frecuencia de las que ven malogrados sus embarazos en el trimesttre intermedio de la gestación, es pequeño, por no decir reducido.

Barter y colaboradores en un estudio conjunto con Parks han encontrado sólo 19 casos en los servicios combinados de tres hospitales, durante un período en el cual atendieron aproximadamente 35.000 pacientes. El total de casos publicados hasta el presente, incluídas las comunicaciones originales de Palmer, no excede en mucho el número de 200.

\section{ETIOLOGIA}

Es un hecho que muchas de las pacientes cuya historia obstétrica sugiere incompetencia cervical no revelan traumatismo o anomalía de éste órgano, cuando se las somete al exámen genital fuera del embarazo. Sin embargo, la mayoría de los autores que se ha ocupado de esta entidad clínica, están de acuerdo en que la incompetencia se debe generalmente a trauma cervical previo, de origen obstétrico ó quirúrgico. La lista de daños revelados en las historias de las pacientes incluye partos difíciles, traumáticos o que han requerido intervención, incisiones cervicales en el momento del parto tipo Dührssen, histerotomias vaginales; conizaciones o amputaciones altas, traquelorrafias, dilataciones y raspados. En mujeres nulíparas la simple dilatación y raspado puede causar incompetencia cervical subsiguiente, especialmente si la operación ha sido prodigada y si las circunstancias sugieren que pudo haber sido un procedimiento traumático. Lash (1) asegura que fué capaz de determinar la causa traumática como origen de incompetencia cervical en todos los 66 casos que ha visto hasta hoy Barter (2) encontró evidencia significativa de trauma obstétrico y quirúrgico en 14 de sus 22 casos.

Según el australiano MacDonald, (4) la historia de sus 70 pacientes reveló una frecuencia anormalmente alta de dilatación operatoria del cuello uterino. Cinco pacientes de su serie, que tuvieron dilatación por dismenorrea, sufrieron una trágica sucesión de abortos entre la décima cuarta y la vegésima octava semanas de la gestación.

\section{DIAGNOSTICO}

Se necesitan dos elementos para establecer el diagnóstico de incompetencia cervical como causa de aborto tardío y parto prema- 
turo; una historia obsttétrica sugestiva y la demostración de un orificio cervical interno dilatado.

tre la $14^{\prime \prime}$ y $28^{\prime \prime}$ semana de: gestación, sin contracciones uterinas

El diagnóstico puede hacerse durante o fuera de la gestación. Si se tiene la oportunidad de estudiar la paciente en caso de amenaza de aborto, la sucesión de los acontecimientos y él hallazgo clínico caracterizado por pérdida repentina de líquido amniótico enprevias, es el hecho más notorio de éstos abortos. en otros casos es posible observar las membranas fetales exteriorizadas a través del orificio cervical interno dilatado. En casos típicos aparecen las membranas sin apariencia de tensión y el cuerpo uterino blando y no irritable. Da la impresión de que la dilatación cervical fuese básicamente un proceso pasivo.

En cada caso se encuentra historia de uno ó varios abortos en el trimestre intermedio de la gestación. Los 22 casos estudiados por Barter (2) habian tenido un total de 91 gestaciones anteriores con sólo 10 niños sobrevivientes.

Fuera de la gestación los hallazgos físicos al examen pélvico no aportan datos de valor, excepto en aquellos casos en los cuales pueden indicar o confirmar el hecho de trauma cervical previo.

La valoración del estado del orificio interno del cuello podría intentarse midiendo su permeabilidad mediante el uso de bujías cervicales graduadas de Hegar. Se ha establecido por autores como Page, (3) Barter (2) y Lash (1) que si el canal cervical permite el paso y la movilización lateral de la bujías Nos. 5 á 8 , sin provocar malestar a las pacientes, es indicio de incompetencia cervical.

Hallazgos más ilustrativos se han ido acumulando en los últimos años, como consecuencia de la introducción de métodos histerográficos simples o complementados con dispositivos especiales, tendientes a observar la silueta producida a nivel del orificio cervical interno. Con el uso de éstos procedimientos se han logrado encontrar defectos del orificio cervical interno puros o asociados con lesiones del canal cervical en el $60 \%$ de pacientes cuyas historias sugerian incompetencia cervical.

\section{TRATAMIENTO}

El objetivo principal del intento terapéutico en este problema ha sido lograr un beneficio duradero mediante refuerzo o reconsttrucción del cuello por medio de procedimientos quirúrgicos. Se han ideado y ensayado algunas técnicas para emplearlas bien durante o fuera de la gestación. En ambas circunstancias el uso de tales métodos ha probado su practicabilidad y eficacia en algunos casos, excepto en los muy raros en los cuales la amputación del cuello ha tan alta como para volver sombrio el futuro obstétrico de la paciente. 
Los primeros esfuerzos encaminados a remediar ésta condición anormal del cuello uterino se deben a Palmer en Francia y Lash (1) en Estados Unidos. Estos investigadores se dieron cuenta de que el defecto principal era una zona débil localizada en las porciones anterior del orificio interno y pared uterina adyacente al cuello.

La técnica correctora de Lash (1) consiste en exponer aquella área, movilizando con tal fin la vejiga a través de incisión transversa de la mucosa vaginal en el limite entre el cuello y vejiga. Luego se extirpa una cuña longitudinal de paredes cervical y miometrial a nivel del orificio interno; la cuña incluye suficiente arco de la luz cervical para reducirla a dimensiones normales, una vez reunidos los bordes mediante sutura. Lash ha operado 66 pacientes con la técnica descrita. Posteriormente informó sobre la suerte de 44 de ellas. De estas, 29 se embarazaron y de ellas, 27 dieron a luz fetos a término. Cuatro de las pacientes requirieron reoperación antes de que pudiera alcanzarse éxito.

El procedimiento de Lash (1) crea una cicatriz vertical en el segmento inferior y en el cuello. La posibilidad de ruptura de ésta cicatriz en las últimas semanas de gestación, causa justa ansiedad y expectativa en la mente del obstetra. Estas pacientes generalmente deben atenderse con operación cesárea. Varios autores han registrado una frecuencia de infertilidad y esterilidad más alta que la normal entre las pacientes operadas con éste método.

En años recientes, la mayoría de los esfuerzos quirúrgicos en relación con la incompetencia cervical se han realizado en pacientes gestentes en el momento en que amenazaban aborto. Cuatro grupos de investigaciones: Shirodkar, Green Armytage (5) y Brown, MacDonald (4) y Barter (2) colaboradores han informado recientemente acerca de sus series de casos operados los cuales varían entre 12 y 70 . La razón por la cual ellos prefieren operar durante la gestación, radica en que durante la década en que se ha venido desarrollando el conocimiento de ésta entidad, ha habido un comprensible acuerdo entre los obstetras referente a un problema que es raro y difícil de diagnósticar con seguridad, excepto en la paciente gestante.

Los signos patognomónicos de la incompetencia cervical sólo se manifiestan típicamente durante el trimestre intermedio del embarazo. De ahí que la mayoría de investigadores prefieren esperar aquel período a fin de establecer el diagnóstico con seguridad y proceder quirúrgicamente. Con una amplia convicción de que muchas de las amenazas de aborto en el segundo trimestre pueden prevenirse con cirugía, hay mayor tendencia a operar inmediatamente a la aparición de los síntomas. Actualmente se considera que la mejor manera de salvar estos fetos se obtiene operando temprano en el segundo trimestre, es decir antes de que la amenaza haya causado dilatación parcial y borramiento del cuello.

Las técnicas operatorias permitidas durante la gestación evidentemente deben limitarse a procedimientos comparativamente simples. 
El método originalmente descrito por Shirodkar utiliza un principio que ha sido adoptado con variaciones por todos los autores. Consiste en la colocación de un torniquete alredero del cuello, debajo de la mucosa, a un nivel tan alto en la porción vaginal del cuello como sea posible. Con tal fin él ha empleado una banda aponeróutica extirpada de la fascia lata de la paciente. E 1 método de Shirodkar ha sido empleado con pequeñas variaciones por Barter (2) y sus colaboradores. Más recientemente ha sido preconizado el uso de Nylon o seda gruesa en lugar de fascia por MacDonald (4) y Green Armytage (5) y Brown, quienes conjuntamente han empleado ésta técnica modificada en 82 operaciones.

Teniendo en cuenta los resultados divulgados por éstos autores, aproximadamente 7 de cada 10 gestaciones, pueden salvarse con los métodos operatorios ordinariamente disponibles para favorecer a la futura madre afligida por incontinencia cervical.

Cuando se usa el procedimiento de Shirodkar, Picot, (6) Thomson y Murphy creen que es innecesario e indeseable esperar hasta que el cervix dilate y exponga las membranas. El procedimiento corrector hecho bajo éstas circunstancias es más difícil técnicamenteHay mayor probalidad de ruptura de membranas, infección amniótica, irritabilidad uterina y aborto. Ellos creen que el ideal es realizar el procedimiento entre la $16^{n}$ y $20^{n}$ semanas.

\section{CONCLUSIONES}

1. Se presenta un caso de aborto habitual por incompetencia cervical comprobada, que logró llevar a término la $4^{*}$ gestación mediante ligadura cervical con la técnica de Shirodkar, modificada por Green Armytage.

2 Se hacen algunas consideraciones etiológicas, clínicas y terapéuticas de la incontinencia cervical.

\section{BIBLIOGRAFIA}

1- Lash, A. F., and Lash, S. R.: Am. J. Obst. and Gynec. $59: 68,1950$.

2- Barter, R. H.: Am. J. Obst. and Gynec. E5:511, 1958.

3- Page, E. W.: Obst. and Gynec. 12:509, 1958.

4- MacDonald, I. A.: J. Obst. and Gynec. Brit. Emp. 64:346, 1957 .

5- Green Armytage, V. B.: Brit. Med. J., 2, 128, 1957.

6- Picot, H.: Am. J. Obst. 78: 786, 1959. 